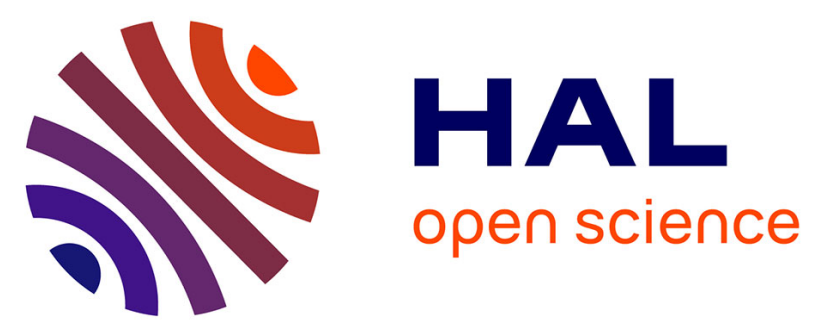

\title{
Remplacement prothétique fémoral total (étiologie tumorale de première intention exclue). Résultats radio-cliniques d'une enquête nationale au recul moyen de 6 ans
}

Sophie Putman, Henri Migaud, Dominique Saragaglia, Jean-Yves Jenny, Franck Dujardin, Antoine-Guy Hue, Fabrice Fiorenza, Olivier Méric, Paul Bonnevialle

\section{- To cite this version:}

Sophie Putman, Henri Migaud, Dominique Saragaglia, Jean-Yves Jenny, Franck Dujardin, et al.. Remplacement prothétique fémoral total (étiologie tumorale de première intention exclue). Résultats radio-cliniques d'une enquête nationale au recul moyen de 6 ans. Revue de Chirurgie Orthopédique et Traumatologique, 2019, 105, pp.407 - 414. 10.1016/j.rcot.2019.03.002 . hal-03486566

\author{
HAL Id: hal-03486566 \\ https://hal.science/hal-03486566
}

Submitted on 20 Dec 2021

HAL is a multi-disciplinary open access archive for the deposit and dissemination of scientific research documents, whether they are published or not. The documents may come from teaching and research institutions in France or abroad, or from public or private research centers.
L'archive ouverte pluridisciplinaire HAL, est destinée au dépôt et à la diffusion de documents scientifiques de niveau recherche, publiés ou non, émanant des établissements d'enseignement et de recherche français ou étrangers, des laboratoires publics ou privés.

\section{(ㄷ)(1) $\$$}

Distributed under a Creative Commons Attribution - NonCommerciall 4.0 International 


\section{Mémoire original}

\section{Remplacement prothétique fémoral total (étiologie tumorale de première intention exclue). Résultats radio-cliniques d'une enquête nationale au recul moyen de 6 ans.}

\section{Total femur replacement in non-oncologic indications: Functional and radiological outcomes from a French survey with a mean 6 years' follow-up}

Sophie Putman ${ }^{a, b, *}$, Henri Migaud ${ }^{\text {a,b }}$, Dominique Saragaglia ${ }^{c}$, Jean-Yves Jenny ${ }^{\mathrm{d}}$, Franck Dujardin ${ }^{\mathrm{e}}$, Antoine-Guy Hue ${ }^{\mathrm{e}}$, Fabrice Fiorenza ${ }^{\mathrm{f}}$ Olivier Méric ${ }^{\mathrm{g}}$, Paul Bonnevialle $^{\mathrm{g}}$, et Société Française de Chirurgie de la Hanche et du Genou (SFHG) ${ }^{\mathrm{h}}$

a Université de Lille Nord de France, 59000 Lille, France

b Service d'Orthopédie, Hôpital Roger Salengro, Place de Verdun, Centre Hospitalier

Régional Universitaire de Lille, 59037 Lille, France

c Département universitaire de chirurgie orthopédique et de traumatologie du sport, CHU de Grenoble Hôpital Sud, Avenue de Kimberley38130, Echirolles, France.

d Centre de Chirurgie Orthopédique et de la Main, Hôpitaux Universitaires de Strasbourg, 10 Avenue Baumann, 67400, Illkirch-Graffenstaden, France.

e Département de chirurgie orthopédique, Hôpital Charles Nicolle, CHU de Rouen, 1 rue de Germont, 76031 Rouen, France

f Service d'Orthopédie Traumatologie CHU Dupuytren, 2, avenue Martin-Luther-King, 87042 Limoges

g Département d'Orthopédie et Traumatologie CHU Toulouse. Hôpital PP Riquet. Place Baylac Toulouse Cedex 31052, France

h 52 rue Boissonade, 75014 Paris, France

* Auteur correspondant : Sophie Putman, Service d'Orthopédie, Hôpital Roger Salengro, Place de Verdun, Centre Hospitalier Régional Universitaire de Lille, 59037 Lille, France 
Mail=sophie.putman@chru-lille.fr

Ne pas utiliser, pour citation, la référence française de cet article, mais celle de l'article original paru dans Orthopaedics \&Traumatology: Surgery \& Research, en utilisant le DOI ci-dessus.

\section{Résumé :}

\section{Introduction}

La prothèse totale de fémur (PTF) a fait l'objet de peu de publications en raison de ses indications exceptionnelles. En dehors des indications tumorales, les indications se sont étendues aux révisions et fractures inter-prothétiques du fémur mais leurs résultats ne sont pas connus avec précision. Cette enquête rétrospective portant sur ces nouvelles indications avait pour objectifs d'analyser les taux de complications, et les résultats fonctionnels.

\section{Hypothèse}

La morbidité et les résultats après PTF sont comparables à ceux de la littérature dans des indications non oncologiques.

\section{Matériel et Méthode}

Entre 1997 et 2016, 29 PTF chez 15femmes et 14 hommes, âgés de 68 ans \pm 14 ans [32-85], issus de $6 \mathrm{CHU}$ ont été implantées. La primo pathologie était 16 arthropathies dégénératives de hanche et/ou du genou, 11 échecs mécaniques de prothèses de résection tumorale et 2 fractures fémorales. Le nombre moyen d'interventions avant la pose de la PTF était de 3,6, (maximum 5) à la hanche et de 4,5 (maximum 10) au genou. Six modèles différents ont été implantés comportant : une charnière rotatoire sauf dans un cas, 9 interfaces coxofémoraux conventionnels et 20 doubles mobilités. La diaphyse fémorale était partiellement conservée $21 / 29$ fois et le trochanter $25 / 29$ fois

\section{Résultats}

Cinq patients ont présenté une complication générale et 12 une complication locale dont 4 hématomes et 2 luxations coxofémorales. Huit patients $(28,6 \%)$ ont présenté une infection du 
site opératoire, mais trois avaient une infection préalable. Parmi les 12 patients présentant un antécédent septique ou une infection évolutive avant la pose de la PTF, 9 ont guéri et 3 sont restés septiques. Au recul minimum de 2 ans et moyen de 6 ans, 23 PTF étaient en place et non septiques et 6 déposées ou septiques dont un patient désarticulé. La médiane de survie des PTF non septiques était de 15 ans ; à 10 ans, 70\% des PTF étaient en place et non septiques.

La marche s'effectuait de manière normale sans canne ou avec une canne chez 15 patients (51,7\%), avec deux cannes ou au déambulateur chez 12 patients (41,3\%); elle était impossible pour deux patients. La flexion active du genou était en moyenne de $79,4^{\circ} \pm 30,3$ [0-120]; 17 patients $(62,9 \%)$ avaient une flexion égale ou supérieure à $90^{\circ}$; deux patients $(7,4 \%)$ n'avaient aucune flexion ; l'extension était déficitaire en moyenne de $3,7^{\circ} \pm 7[-20$ à 10]: 20 patients n'avaient aucun flexum. L'inégalité moyenne finale était de 1,3 $\mathrm{cm} \pm 2,3$ [010]; 19 patients $(67,8 \%)$ étaient à l'équilibre.

\section{Discussion}

L'hypothèse de départ est confirmée en comparant le taux de complication et les résultats cliniques. L'intérêt des cupules double mobilité est souligné. Les indications restent exceptionnelles mais en probable augmentation dans les années à venir.

Niveau de preuve : IV, étude de cohorte rétrospective

Mots clefs: Arthroplastie totale de fémur. Fracture fémorale inter prothétique Descellement itératif arthroplastie de hanche. Descellement itératif arthroplastie de genou. Infection chronique prothétique. 


\section{Introduction}

La prothèse totale de fémur (PTF) - association d'une arthroplastie de hanche (PTH) et de genou (PTG) reliées par un implant diaphysaire - a été réalisée pour une diffusion sarcomateuse par Marcove et al. [1] puis validée en chirurgie oncologique [2-6]. Buchman avait précédemment publié un cas de résection-remplacement pour fémur pagétique [7] suivi de quelques publications dans le même contexte non tumoral [8-11]. La place de ces implants est limitée, illustrée par la rareté des publications et le nombre de cas rapportés en dehors de Berend et al. [12] et Friesecke et al. [13].

Dans la littérature française, Soenen et al. [14] ont rapporté 3 PTF pour des échecs itératifs de fractures inter-prothétiques. En dehors des résections tumorales, les indications de PTF demeurent exceptionnelles s'adressant aux reprises septiques et mécaniques multi opérées ainsi qu'aux échecs mécaniques de prothèses de résection tumorale [15-19]. Leurs résultats ne sont pas connus dans ces indications notamment en France. Une enquête rétrospective auprès des membres de la Société française de la hanche et du genou (SFHG) a donc été menée, dans ces nouvelles indications afin d'analyser 1) les taux de complications, 2) et les résultats fonctionnels. Notre hypothèse était que la morbidité et les résultats après PTF sont comparables à ceux de la littérature dans des indications non oncologiques.

\section{Matériel et méthode}

\subsection{Critères d'inclusion}

Les critères d'inclusion étaient les échecs mécaniques itératifs d'une PTH et /ou d'une PTG ipsilatérale émaillés ou non de fracture intra ou inter prothétique, une cause septique avec dépose-repose en un ou deux temps. Les échecs d'arthroplasties (PTF, PTH ou PTG) implantées pour une étiologie tumorale chez un patient en longue rémission ou guéri ont été 
inclus. Un recul minimum de 2 ans a été retenu. Le critère d'exclusion était toute PTF posée en première intention pour résection tumorale et/ou au recul inférieur à 2 ans.

\subsection{Patients}

Entre 1997 et 2016, 29 patients issus des CHU de Grenoble ( $n=4)$, Lille ( $n=9)$, Limoges $(n=1)$, Rouen $(n=4)$, Strasbourg $(n=4)$ et Toulouse $(n=7)$ ont eu une PTF. Il s'agissait de 15 femmes et 14 hommes, âgés de 68 ans \pm 14 ans [32-85] à la pose. Trois patients étaient ASA 1, 17 patients ASA 2, 8 patients ASA 3 et 1 patient ASA 5 [20]. Le score moyen de Parker et Palmer préopératoire était de 5,4 [0-9] [21].

La pathologie fémorale initiale était une fracture dans deux cas, une résection tumorale dans 11 cas et l'indication était alors un échec mécanique sur un malade guéri sur le plan carcinologique et 16 cas d'arthropathie dégénérative de hanche et/ou du genou. Pour les 11 patients répondant à une étiologie tumorale, le recul moyen était 12,4 ans extrêmes 3 et 23 ; aucun ne présentait de signe clinique et /ou radiologique d'évolutivité locale. L'histoire de ces 29 cas est détaillée dans la figure 1. Vingt-sept des 29 cas avaient eu plusieurs interventions avant la pose du fémur total et les histoires cliniques sont détaillées dans la figure 2. Le nombre moyen d'interventions par patient toute localisation confondue avant la pose de la PTF était de 3,6 (1 à 10). A la hanche le nombre était de 3,6 $\pm 1,8$ et maximum 5 et au genou de $4,5 \pm 2,4$, maximum 10 . Les motifs ayant conduit à l'implantation d'un fémur total sont détaillés dans la figure 3.

A la pose de la PTF, 12 patients avaient un antécédent septique sur le fémur opéré au niveau au moins d'une des deux arthroplasties dont 8 en infection évolutive. Trois étaient porteurs d'une arthroplastie de hanche et de genou et 9 avaient présenté une fracture périprothétique sur PTH ou PTG. 


\subsection{Technique opératoire}

Les modèles implantés étaient 10 Méga System (Waldemark- Link, Hambourg, Germany), 7 Global Modular Replacement System (Stryker, Pusignan, France) (Figure 4), 7 Mutars (Implantcast GmBH, Buxtehude, Germany) (Figure 5), 2 Modular Endoprosthetic Total System (Stanmore, Borehamwood, UK), 2 Orthopaedics Salvage System (Zimmer-Biomet, Brognard, France) et 1 Askorn (Askorn Medical, Cesson-Sévigné, France). Les interfaces coxofémoraux étaient 9 fois conventionnels et 20 fois en double mobilité. Dans 6 cas, une armature métallique acétabulaire a été utilisée. Au genou, les charnières étaient toutes rotatoires sauf pour 1 implant. La diaphyse fémorale a été partiellement conservée 21 fois et totalement réséquée 8 fois ; pour le trochanter il y a eu 25 conservations et 4 ablations. Dans 6 cas, une armature métallique acétabulaire a été utilisée. Tous les implants tibiaux étaient cimentés.

\subsection{Méthode d'évaluation}

La saisie des dossiers a été faite sur un tableur rassemblant les items épidémiologiques conventionnels et en particulier les scores pré- et postopératoires de l'ASA [20] et de Parker et Palmer [21] pour les patients de plus de 65 ans. L'évaluation radio clinique s'est faite au plus long recul connu, au-delà de la deuxième année.

\subsection{Méthodes statistiques}

Les tests statistiques ont été réalisés par le logiciel Statis (8.4.6. PC O. Méricq. Toulouse). Les variables qualitatives ont été décrites par les effectifs et les pourcentages et les variables numériques par la moyenne et l'écart-type. Ont été utilisés le test paramétrique de Student pour la comparaison des différentes moyennes et le test de $\mathrm{Chi}^{2}$ pour les variables qualitatives 
indépendantes lorsque l'effectif des groupes était supérieur à 5. Pour des effectifs inférieurs à 5, le test exact de Fisher a été utilisé. Le seuil de significativité a été fixé à p< 0,05.

\section{Résultats}

\subsection{Complications}

Un patient a eu une embolie graisseuse per-opératoire avec arrêt cardio-respiratoire régressif. En postopératoire précoce, quatre patients ont eu une complication générale : 1 accident vasculaire cérébral laissant des séquelles neurologiques, une embolie pulmonaire et 2 insuffisances rénales liées aux antibiotiques. Les complications locales ont été 4 hématomes, 2 luxations coxofémorales, 3 incidents cicatriciels, 1 désassemblage prothétique, un arrachement du trochanter, et une avulsion de la tubérosité tibiale antérieure. Huit patients $(27,6 \%)$ ont eu une infection du site opératoire (trois étaient porteurs d'une infection évolutive). Parmi les 12 patients présentant un antécédent septique ou une infection évolutive avant la pose de la PTF, 9 ont guéri et 3 sont restés septiques, dont un patient nécessitant une désarticulation Les quatre cas d'étiologie exclusivement septique rapportés par l'un de nous (JYJ) ont tous évolué favorablement.

\subsection{Survie}

Le recul minimum était de 2 ans, moyen de $6 \pm 4,5$ ans. Dans notre série, les patients avec un antécédent septique avaient le plus long recul. La médiane de survie actuarielle était de 18,1 ans ; à 10 ans $59 \%$ des patients étaient en vie. Au plus long recul, 23/29 (79,6\%) PTF étaient en place et non septiques et $6(20,6 \%)$ déposées ou septique sous antibiothérapie suppressive. La médiane de survie des 17 PTF sans antécédents septiques était de 15 ans ; à 10 ans, 12/17 (70\%) des PTF sans antécédents septiques étaient en place et non septiques.

\subsection{Résultats fonctionnels}


La marche s'effectuait de manière normale sans canne chez 5 patients $(17,2 \%)$, avec une canne chez 10 patients $(34,5 \%)$, avec deux cannes chez 7 patients $(24,1 \%)$ et avec un déambulateur chez 5 patients $(17,2 \%)$. Elle était impossible pour deux patients $(6,9 \%)$ dont le patient désarticulé. La flexion active du genou (évaluée chez 27 patients) était en moyenne de $79,4^{\circ} \pm 30,3^{\circ}\left[0^{\circ}-120^{\circ}\right] ; 17$ patients $(62,9 \%)$ avaient une flexion égale ou supérieure à $\left.90^{\circ}\right), 8$ patients $(29,6 \%)$ avaient une flexion comprise entre 40 et $85^{\circ}$ et deux patients $(7,4 \%)$ n'avaient aucune flexion. L'extension du genou était déficitaire de $3,7 \pm 7^{\circ}[-20,+10]$ en moyenne, maximum de $20^{\circ}: 20$ patients $(74,1 \%)$ n'avaient aucun flexum. L'inégalité moyenne finale des membres inférieurs était de $1,3 \pm 2,3 \mathrm{~cm}(0$ à $10 \mathrm{~cm}) ; 19$ patients $(67,8 \%)$ étaient à l'équilibre à $1 \mathrm{~cm}$ près. Le score de Parker et Palmer final moyen était de 6,1 $\pm 1,9 ; 6$ patients avaient un score supérieur ou égal à $8(25 \%)$ et 5 un score entre 1 et 2 $(20,8 \%)$.

\section{Discussion}

Trois principaux enseignements peuvent être tirés de cette série: 1) la rareté des indications et leur caractère exceptionnel soit 29 dossiers répartis entre 6 services sur une période de 20 ans. Mais leur fréquence va sans doute augmenter avec les révisions de prothèses tumorales et le vieillissement de la population favorisant les reprises des fractures inter-prothétiques sur des os fragiles. 2) la fréquence des complications septiques (28\%) est à souligner dont un cas a conduit à une désarticulation. 3) enfin le caractère modeste des résultats cliniques est évident : la marche était normale ou avec une canne chez seulement 15 patients $(58,6 \%)$,

Parmi les 6 séries publiées de PTF pour étiologie non oncologique, deux études monocentriques se distinguent par leur importance numérique (Tableau 1). Berend et al. [13] rapportent les résultats d'une série de 59 patients opérés d'une fracture péri-prothétique ou de 
descellement itératifs. La série de l'Endo-Klinik de Hambourg rapportée par Friesecke et al. [14] est encore plus fournie rassemblant 100 patients consécutifs opérés entre 1973 et 2000. Les indications de la présente série sont globalement identiques à celles de la littérature quant à l'âge, et le nombre d'interventions préalables démontrant le caractère extrême de l'indication de la PTF. Il en est de même des résultats cliniques, partiellement comparables en raison des critères d'évaluation retenus montrant essentiellement une amélioration de l'autonomie des patients.

La rareté des luxations postopératoires de cette cohorte $(2 / 29$ soit $6,9 \%)$ est à mettre au crédit de l'utilisation quasi systématique d'implants acétabulaires en double mobilité alors que le taux d'instabilité prothétique est de 25 à $40 \%$ dans certaines séries (Tableau 2). Les implants double mobilité ont démontré tout leur intérêt dans le contexte des résections tumorales [2224]. En effet le risque d'instabilité est élevé en raison de l'amyotrophie glutéale secondaire aux interventions multiples. La résection diaphysaire imposant une réinsertion directe et aléatoire des muscles sur l'implant et la valeur mécanique incertaine de la fixation du trochanter voire sa résection augmente ce risque d'instabilité. Dans la plupart des cas de notre série, la conservation osseuse bien que plus exigeante [25-26] était recherchée lorsque celle-ci était possible (21 conservations de diaphyses, 25 conservations du massif trochantérien).

Le taux d'infection postopératoire de cette série se situe dans la fourchette de celui rapporté dans la littérature (Tableau 2). Il correspond à celui des méga prothèses en chirurgie de résection carcinologique [27] Le taux maximum d'infection vient de la seule série ayant un recul minimum de 2 ans [19] à l'instar des autres études rapportées. Comme dans la présente série, un recul de plus de 2 ans est recommandé pour affirmer une évolution non septique d'une révision d'arthroplastie et ce d'autant qu'existe un antécédent septique ou en présence d'une infection avérée. 
L'indication de PTF a été posée dans cette série chez 12 patients (41\%) ayant un antécédent septique ou face à une infection présente : ce taux est proche de ceux de la littérature (Tableau 3). Dans les séries de Berend et al. [12] et de Frisecke et al. [13] le taux de guérison septique après la pose de la PTF est plus élevé que dans notre série mais avec un recul inférieur. Les quatre cas d'étiologie exclusivement septique rapportés par l'un de nous (JYJ) ont tous évolué favorablement confirmant l'intérêt d'une dépose-repose en un temps dans un centre de référence [28,29].

Les limites de cette étude sont importantes et liées en particulier à la diversité des origines des dossiers, le caractère multi opérateur, l'étalement dans le temps et l'utilisation d'implants différents. Cependant, l'implantation quasi systématique d'une charnière rotatoire au genou et l'utilisation très fréquente d'un implant coxo-fémoral à double mobilité sont des facteurs d'homogénéité. De même, le caractère extrême des interventions et le nombre élevé d'interventions et d'infections préalables permet d'avoir une idée de cette procédure dans les pires conditions augmentant la validité externe des conclusions.

\section{Conclusion}

Cette série tant par ses indications que par les résultats cliniques et les complications constatées est comparable à la littérature et confirme notre hypothèse de départ. Les indications d'une PTF sont exceptionnelles mais avec l'augmentation du nombre de patients porteurs d'une arthroplastie de hanche et de genou homolatérale mécaniquement défaillante et/ou septique, cette circonstance sera en augmentation dans l'avenir. Poser cette indication c'est aussi se rappeler du risque de désarticulation en cas d'échec.

\section{Conflit d'intérêt.}


Aucun des auteurs ne déclare de liens en relation avec ce travail. En dehors de ce travail Paul Bonnevialle est consultant recherche et enseignement pour Stryker, Sophie Putman est consultant éducation et recherche pour Corin-Tornier, Henri Migaud est rédacteur associé pour Orthopaedics \& Traumatology Surgery \& Research et consultant éducation et recherche pour Zimmer-Biomet, Corin-Tonier MSD et SERF, JY Jenny déclare des royalties de B-Braun Aesculap, a été un consultant rémunéré d' Exatech et a reçu des soutiens pour congrès de FH Orthopadics, D. Saragaglia déclare des royalties de B-Braun Aesculap, F. Dujardin est consultant pour SERF. Les autres auteurs ne déclarent pas de conflits en dehors de ce travail.

\section{Financement}

Pas de financement

\section{Contributions}

PB a collecté les données, opéré les patients et rédigé l'article. HM et SP ont opéré les patients, collecté les données, et rédigé l'article ; DS JYJ FD et FF ont opéré les patients et collecté les données. AGH et OM ont collecté les données. 
Tableau 1 : Tableau comparatif démographique des principales séries récentes de la littérature comportant au moins 10 patients. $\mathrm{NC}=$ non communiqué. (dans les séries 13 et 15

respectivement 4 et 2 patients présentaient une étiologie métastatiques).

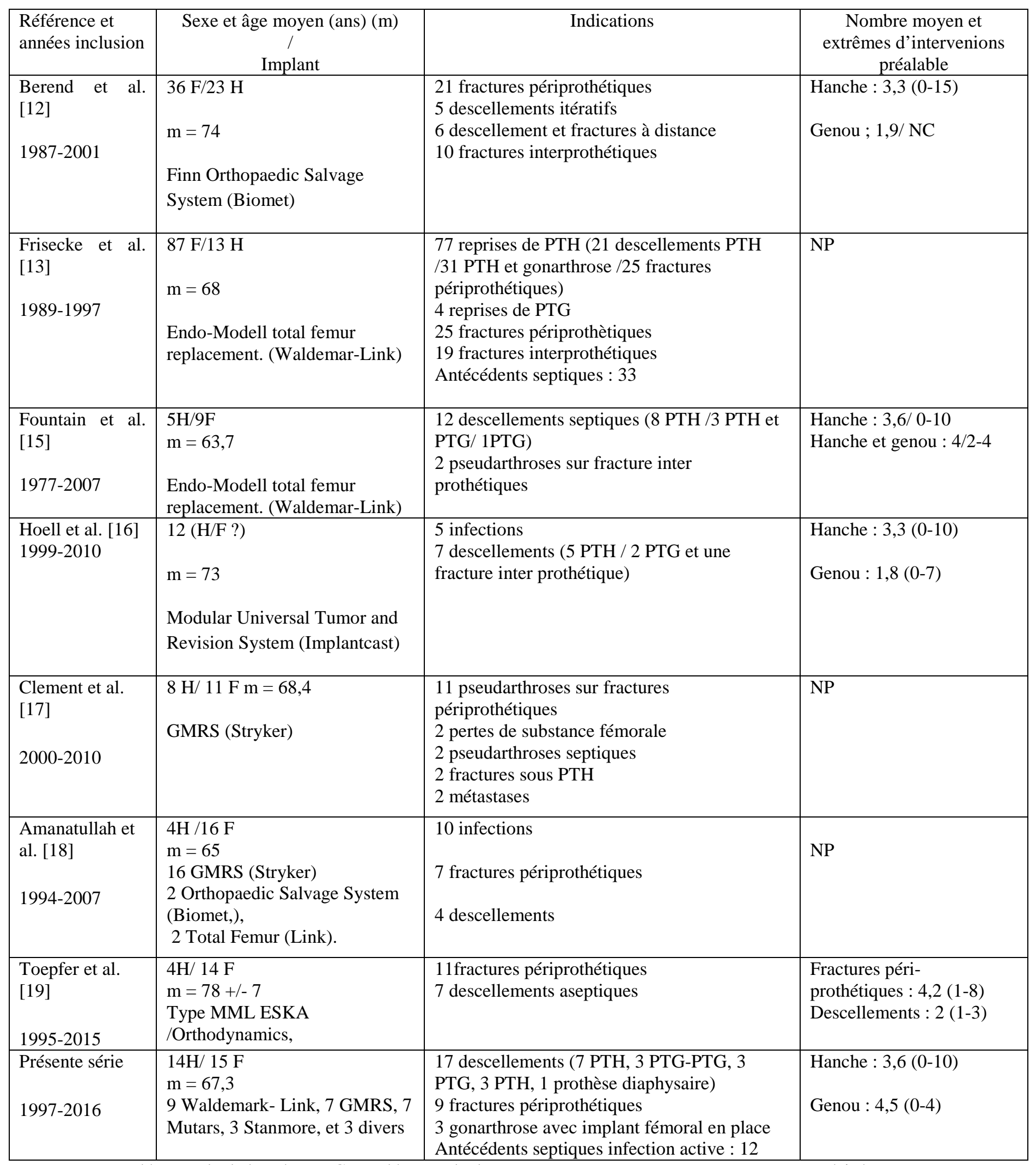

PTH: Prothèse totale de hanche, PTG: prothèse totale de genou, H : Homme, F: Femme, NP : Non précisé 
Tableau 2: Tableau comparatif des résultats des principales séries récentes de la littérature.

\begin{tabular}{|c|c|c|c|c|c|}
\hline Référence & $\begin{array}{c}\text { Reculs } \\
\text { extrêmes et } \\
\text { moyen }\end{array}$ & $\begin{array}{c}\text { Complications } \\
\text { Septiques post } \\
\text { opératoires }\end{array}$ & Autres complications & $\begin{array}{c}\text { Survie } \\
\text { implant }\end{array}$ & Nombre de décès \\
\hline $\begin{array}{l}\text { Berend et al. } \\
\text { [12] }\end{array}$ & $\begin{array}{c}1-13 \text { ans } \\
\mathrm{m}=4 \text { ans } \\
10 \text { mois }\end{array}$ & $8 / 59(13,5 \%)$ & $\begin{array}{l}5 \text { luxations }(8,4 \%) \\
2 \text { inégalités longueur } \\
1 \text { raideur genou }\end{array}$ & $\begin{array}{c}75 \% \text { à } \\
12,5 \text { ans }\end{array}$ & $\begin{array}{c}14 \\
(23,7 \%) \\
\text { Aucun en périopératoire }\end{array}$ \\
\hline $\begin{array}{l}\text { Frisecke et al. } \\
{[13]}\end{array}$ & $\begin{array}{l}1 \mathrm{mois}- \\
11,5 \text { ans } \\
\mathrm{m}=4,9 \\
\text { ans }\end{array}$ & $\begin{array}{l}12 / 100(12 \%) \\
\text { (6 précoces / } 6 \\
\text { tardives) : }\end{array}$ & $\begin{array}{l}7 \text { luxations (7\%) } \\
3 \text { descellements }\end{array}$ & NP- & $\begin{array}{c}3 \text { en périopératoire } \\
(3 \%)\end{array}$ \\
\hline $\begin{array}{l}\text { Fountain et al. } \\
\text { [15] }\end{array}$ & $\begin{array}{l}1 \text { à17 ans } \\
\mathrm{m}=7,5 \\
\text { ans }\end{array}$ & $\begin{array}{l}3 / 14 \text { infections }(21 \%) \\
2 \text { profondes } \\
1 \text { superficielle }\end{array}$ & $\begin{array}{l}6 \text { luxations }(42,8 \%) \\
3 \text { reprises avec succès/ } 3 \\
\text { laissées luxées }\end{array}$ & NP & 1 à 1 an \\
\hline Hoell et al. [16] & $\begin{array}{l}0,5 \text { à } 7,5 \\
\quad \text { ans } \\
\mathrm{m}=2,6\end{array}$ & Aucune & 1 paralysie sciatique & $\mathrm{NP}$ & Aucun \\
\hline $\begin{array}{l}\text { Clement et al. } \\
\text { [17] }\end{array}$ & $\begin{array}{c}0,7-12,4 \\
\mathrm{~m}=5,4\end{array}$ & $\begin{array}{l}\text { 2/19 (10,5\%): } \\
\text { - antibiothérapie } \\
\text { suppressive } \\
\text { - désarticulation }\end{array}$ & $\begin{array}{l}1 \text { luxation (réduction / } \\
\text { succès) }\end{array}$ & $\begin{array}{l}86 \% \text { (à } \\
10 \text { ans) }\end{array}$ & $\begin{array}{l}5 \% \text { à } 1 \text { an } \\
58 \% \text { à } 10 \text { ans }\end{array}$ \\
\hline $\begin{array}{l}\text { Amanatullah et } \\
\text { al. [18] }\end{array}$ & $\begin{array}{c}\mathrm{m}=6 \pm 5 \\
\text { minimum2 } \\
\text { ans }\end{array}$ & $7 / 20(35 \%)$ & $\begin{array}{l}5 \text { luxations }(25 \%) \text { : } \\
-4 \text { révisés } \\
-1 \text { réduction (succès) }\end{array}$ & $\begin{array}{l}\text { 65\% (à } 5 \\
\text { ans) }\end{array}$ & Aucun \\
\hline $\begin{array}{l}\text { Toepfer et al. } \\
\text { [19] }\end{array}$ & $\begin{array}{l}\mathrm{m}=6,6 \\
(2,3-11)\end{array}$ & $8 / 18(44,4 \%)$ & $\begin{array}{l}5 \text { luxations }(27,7 \%) \\
2 \text { raideurs } \\
11 \text { incidents cicatriciels }\end{array}$ & $\begin{array}{c}56 \% \text { (à } \\
5 \text { ans) }\end{array}$ & Aucun \\
\hline Présente série & $\begin{array}{l}\mathrm{m}=6 \\
(2-10)\end{array}$ & $8 / 29(27,6 \%)$ & $\begin{array}{l}2 \text { luxations }(6,9 \%) \\
4 \text { hématomes } \\
3 \text { incidents cicatriciels }\end{array}$ & $\begin{array}{l}70 \% \text { à } 10 \\
\text { ans }\end{array}$ & $\begin{array}{c}41 \% \text { des patients décédés } \\
\text { à } 10 \text { ans }\end{array}$ \\
\hline
\end{tabular}


Tableau 3: Tableau comparatif focalisé sur les complications septiques et les étiologies septiques des principales séries récentes de la littérature

\begin{tabular}{|c|c|c|}
\hline Reference & $\begin{array}{c}\text { Complications } \\
\text { Septiques post opératoires et devenir }\end{array}$ & $\begin{array}{l}\text { Patients à antécédent septique /série } \\
\text { complète. Devenir }\end{array}$ \\
\hline Berend et al. [12] & $\begin{array}{l}\text { 8/ } 49(16,3 \%) \text { : } \\
-3 \text { déposes reposes (succès) } \\
\text { - } 1 \text { échec (désarticulation) } \\
-4 \text { NP }\end{array}$ & $\begin{array}{l}\text { 14/ } 49(23,7 \%): \\
-1 \text { récidive } \\
\text { (désarticulation) } \\
-13 \text { guérisons }(92,8 \%)\end{array}$ \\
\hline Frisecke et al. [13] & $\begin{array}{l}\text { 12/100 (12\%) } \\
\text { - } 2 \text { échecs (désarticulation) } \\
-10 \text { guérisons }\end{array}$ & $\begin{array}{l}33 / 100(33 \%): \\
-5 \text { récidives } \\
-28 \text { guérisons }(84,8 \%)\end{array}$ \\
\hline Fountain et al. [15] & $\begin{array}{l}3 / 14(21,4 \%) \text { : } \\
-1 \text { évolution favorable sous } \\
\text { antibiothérapie } \\
\text { - } 2 \text { reprises en } 2 \text { temps / Guéries }\end{array}$ & $\begin{array}{l}12 / 14(85,7 \%) \\
\text { Devenir NP }\end{array}$ \\
\hline Hoell et al. [16] & $0 / 12$ & $\begin{array}{l}5 / 12(41,6 \%): \\
5 \text { guérisons }(100 \%)\end{array}$ \\
\hline Clement et al. [17] & $\begin{array}{l}\text { 2/19 }(10,5 \%): \\
-1 \text { antibiothérapie suppressive } \\
-1 \text { désarticulation }\end{array}$ & $\begin{array}{l}2 / 19(10,5 \%): \\
2 \text { échecs (idem infection post } \\
\text { opératoire) }\end{array}$ \\
\hline Amanatullah et al. [18] & $\begin{array}{l}\text { 7/20: } 5 \text { reprises NP dont } \\
\text { désarticulation/ Changement en } 1 \text { ou } 2 \\
\text { temps }\end{array}$ & $10 / 20(50 \%):$ devenir NP \\
\hline Toepfer et al. [19] & $\begin{array}{l}8 / 18(44,4 \%): 8 \text { guérisons }(1 \text { sans } \\
\text { changement d'implant, } 4 \text { partielles et } 3 \\
\text { complètes) }\end{array}$ & Aucun cas septique \\
\hline Présente série & $\begin{array}{l}8 / 29(27,6 \%): 5 \text { guérisons / } 3 \text { échecs ( } 3 \\
\text { antibiothérapie suppressive) }\end{array}$ & $\begin{array}{l}\text { 12/ } 29(41,4 \%): \\
3 \text { infections chroniques } \\
9 \text { guérisons }(75 \%)\end{array}$ \\
\hline
\end{tabular}




\section{References}

1/ Marcove R, Lewis MM, Rosen R, Huvos A. Total femur and total knee replacement. A preliminary report. Clin Orthop Relat Res 1977;126:47-52.

2/ Katznelson A, Nerubay J. Total femur replacement in sarcoma of the distal end of the femur. Acta Orthop Scand 1980;51:845-51.

3/ Nerubay J, Katznelson A, Tichler T, Rubinstein Z, Morag B, Bubis JJ. Total femoral replacement Clin Orthop Relat Res 1988;229:143-8.

4/ Sewell MD1, Spiegelberg BG, Hanna SA, Aston WJ, Bartlett W, et al. Total femoral endoprosthetic replacement following excision of bone tumours. J Bone Joint Surg Br 2009;91:1513-20.

5/ Kalra S, Abudu A, Murata H, Grimer RJ, Tillman RM, Carter SR. Total femur replacement: primary procedure for treatment of malignant tumors of the femur. Eur J Surg Oncol 2010; 36:378-83.

6/ Sevelda F, Schuh R, Hofstaetter JG, Schinhan M, Windhager R, Funovics PT. Total femur replacement after tumor resection: limb salvage usually achieved but complications and failures are common. Clin Orthop Relat Res 2015;473:2079-87.

7/ Buchman J. Total femur and knee joint replacement with a vitallium endoprosthesis. Bull Hosp Joint Dis 1965;26:21-34.

8/ Huang G, Cao M. Total replacement of the femur with simultaneous total hip and knee joint endoprostheses with plasma sprayed ceramic coating. Zentralbl Chir 1991;116:199-207. 
9/ Ward WG, Dorey F, Eckardt JJ. Total femoral endoprosthetic reconstruction. Clin Orthop Relat Res 1995;316:195-206.

10/ Freedman EL1, Eckardt JJ. A modular endoprosthetic system for tumor and non-tumor reconstruction: preliminary experience. Orthopedics 1997;20:27-36.

11/ McLean A, Patton JT, Moran M. Femoral replacement for salvage of periprosthetic fracture around a total hip replacement. Injury 2012;43:1166-9.

12/ Berend K, Lombardi A, Mallory T, Adams J, Dodds K. Total femoral arthroplasty for salvage of end-stage prosthetic disease. Clin Orthop Relat Res 2004;427:162-70.

13/ Friesecke C1, Plutat J, Block A. Revision arthroplasty with use of a total femur prosthesis. J Bone Joint Surg Am 2005;87:2693-701.

14/ Soenen M, Migaud H, Bonnomet F, Girard J, Mathevon H, Ehlinger M. Interprosthetic femoral fractures: analysis of 14 cases. Proposal for an additional grade in the Vancouver and SoFCOT classifications. Orthop Traumatol Surg Res 2011;97:693-8.

15/ Fountain JR1, Dalby-Ball J, Carroll FA, Stockley I. The use of total femoral arthroplasty as a limb salvage procedure: the Sheffield experience. J Arthroplasty 2007;22:663-9.

16/ Hoell S, Butschek S, Gosheger G, Dedy N, Dieckmann R, et al. Intramedullary and total femur replacement in revision arthroplasty as a last limb-saving option: is there any benefit from the less invasive intramedullary replacement? J Bone Joint Surg Br 2011;93:1545-9.

17/ Clement N, MacDonald D, Ahmed I, Patton J, Howie C. Total femoral replacement for salvage of periprosthetic fractures. Orthopedics 2014;37:789-95. 
18/ Amanatullah D, Trousdale R, Hanssen A, Lewallen D, Taunton M. Non-oncologic total femoral arthroplasty: retrospective review. J Arthroplasty 2014;29:2013-5.

19/ Toepfer A, Harasser N, Petzschner I, Pohlig F, Lenze U, et al. Short to long term follow-up total femoral replacement in non oncologic patients. BMC Musculoskeletal disorders 2016;16:498-507.

20/ ASA Physical Classification System, http://www.asah-q.org/Home/For-Members/ClinicalInformation/ASA-Physical-Status-Classification-System

21/ Parker MJ, Palmer CR. A new mobility score for predicting mortality after hip fracture J Bone Joint Surg Br 1993;75:797-8.

22/ Philippot R, Adam P, Reckhaus M, Delangle F, Verdot F, Curvale G, Farizon F. Prevention of dislocation in total hip revision surgery using a dual mobility design. Orthop Traumatol Surg Res 2009;95:407-13.

23/ Philippeau JM, Durand JM, Carret JP, Leclercq S, Waast D, Gouin F. Dual mobility design use in preventing total hip replacement dislocation following tumor resection. Orthop Traumatol Surg Res 2010;96:2-8.

24/ Schneider L, Philippot R, Boyer B, Farizon F. Revision total hip arthroplasty using a reconstruction cage device and a cemented dual mobility cup. Orthop Traumatol Surg Res 2011;97:807-13.

25/ Lombardi AV Jr1, Berend KR. The shattered femur: radical solution options. J Arthroplasty. 2006 Jun;21(4 Suppl 1):107-11. 
26/ Peters CL1, Hickman JM, Erickson J, Lombardi AV, Berend KR, Mallory TH.Intramedullary total femoral replacement for salvage of the compromised femur associated with hip and knee arthroplasty. J Arthroplasty. 2006 Jan;21(1):53-8.

27/ Mavrogenis AF, Pala E, Angelini A, Calabro T, Romagnoli C, et al. Infected prostheses after lower-extremity bone tumor resection: clinical outcomes of 100 patients. Surg Infect 2015;16:267-75.

28/ Jenny JY, Lengert R, Diesinger Y, Gaudias J, Boeri C, Kempf JF. Routine one-stage exchange for chronic infection after total hip replacement. Int Orthop 2014;38:2477-81.

29/ Massin P, Delory T, Lhotellier L, Pasquier G, Roche O, et al. Infection recurrence factors in oneand two-stage total knee prosthesis exchanges. Knee Surg Sports Traumatol Arthrosc 2016;24:3131- 


\section{Légendes des figures}

Figure 1: Diagramme de flux détaillant les pathologies initiales des 29 patients ayant eu'un fémur total (PTH : prothèse totale de hanche, PTG : prothèse totale de genou),

Figure 2: Diagramme de flux détaillant les interventions itératives avant l'implantation du remplacement fémoral total (PTF : prothèse de fémur total, PTH : prothèse totale de hanche, RePTH : révision de prothèse totale de hanche),

Figure 3 : Flowchart détaillant les motifs d'implantation du remplacement fémoral total (PTF)(PTH : prothèse totale de hanche, PTG : prothèse totale de genou),

Figure 4 : Patiente âgée de 70 ans, marche en déambulateur, descellement bipolaire de PTG (première pose en 1992, descellement en 2000 traitée par charnière) et de PTH (pose en 1985), fracture itérative inter prothétique (4A et 4B). 4C. Implant GMRS posé en 2012. Résultat clinique (2017) : marche une canne, genou $0 / 90^{\circ}$, inégalité de longueur de $3 \mathrm{~cm}$ compensée, Parker 7.

Figure 5 : Patient âgé de 47 ans. Résection distale du fémur en 1990 pour ostéosarcome avec prothèse de reconstruction, révisée en 2003 (simple reprise cimentée fémorale sans reconstruction) puis perdu de vue jusqu'en 2013 où il consulte pour raideur (20 degrés de flessum $/ 90^{\circ}$ de flexion) avec des douleurs importantes et un raccourcissement de $4,5 \mathrm{~cm}$. 5A : Le capital osseux résiduel est modeste et le risque de fausse route ainsi que les difficultés d'extraction du ciment ont conduit à un remplacement fémoral total (prothèse Mutars) ayant un très bon résultat au recul de 4 ans $(5 \mathrm{~B})$ (pas de douleurs, pas de boiterie, marche sans canne et flexion de genou $95^{\circ}$ avec $10^{\circ}$ de flessum et correction de l'inégalité de longueur à $5 \mathrm{~mm}$ près ne justifiant pas de compensation). Le massif trochantérien et l'os proximal résiduel ont été conservés favorisant la stabilité du bassin. 


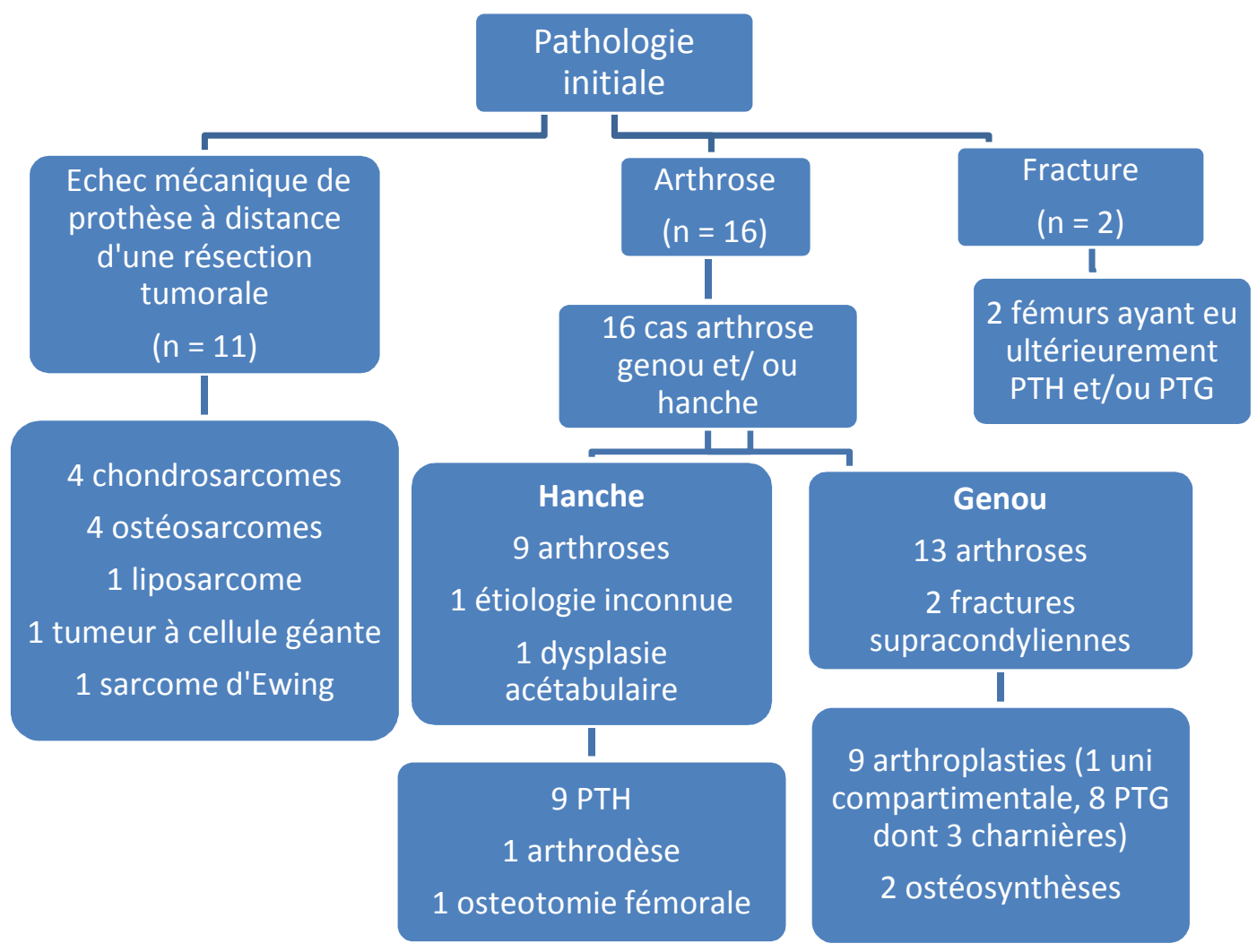




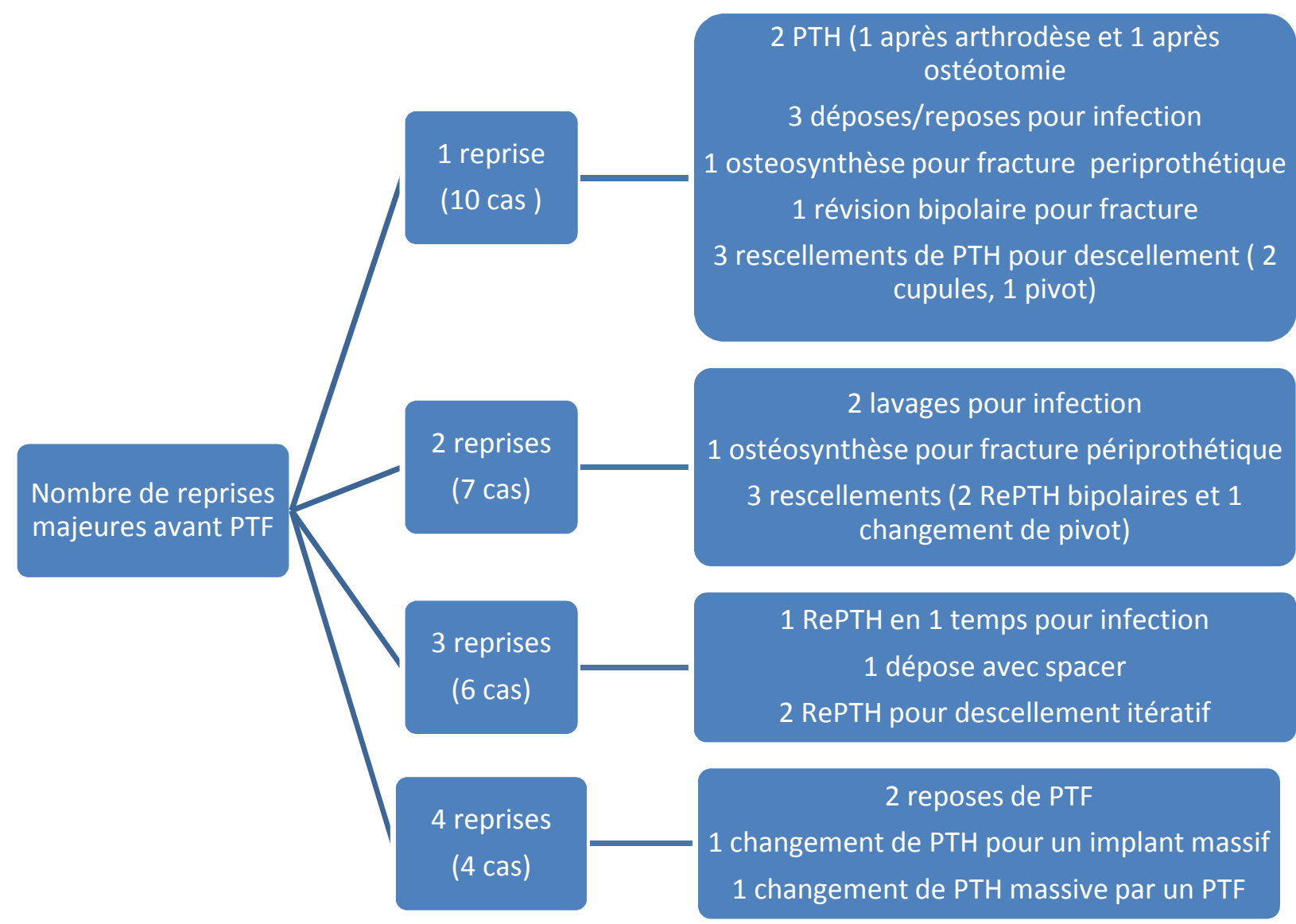




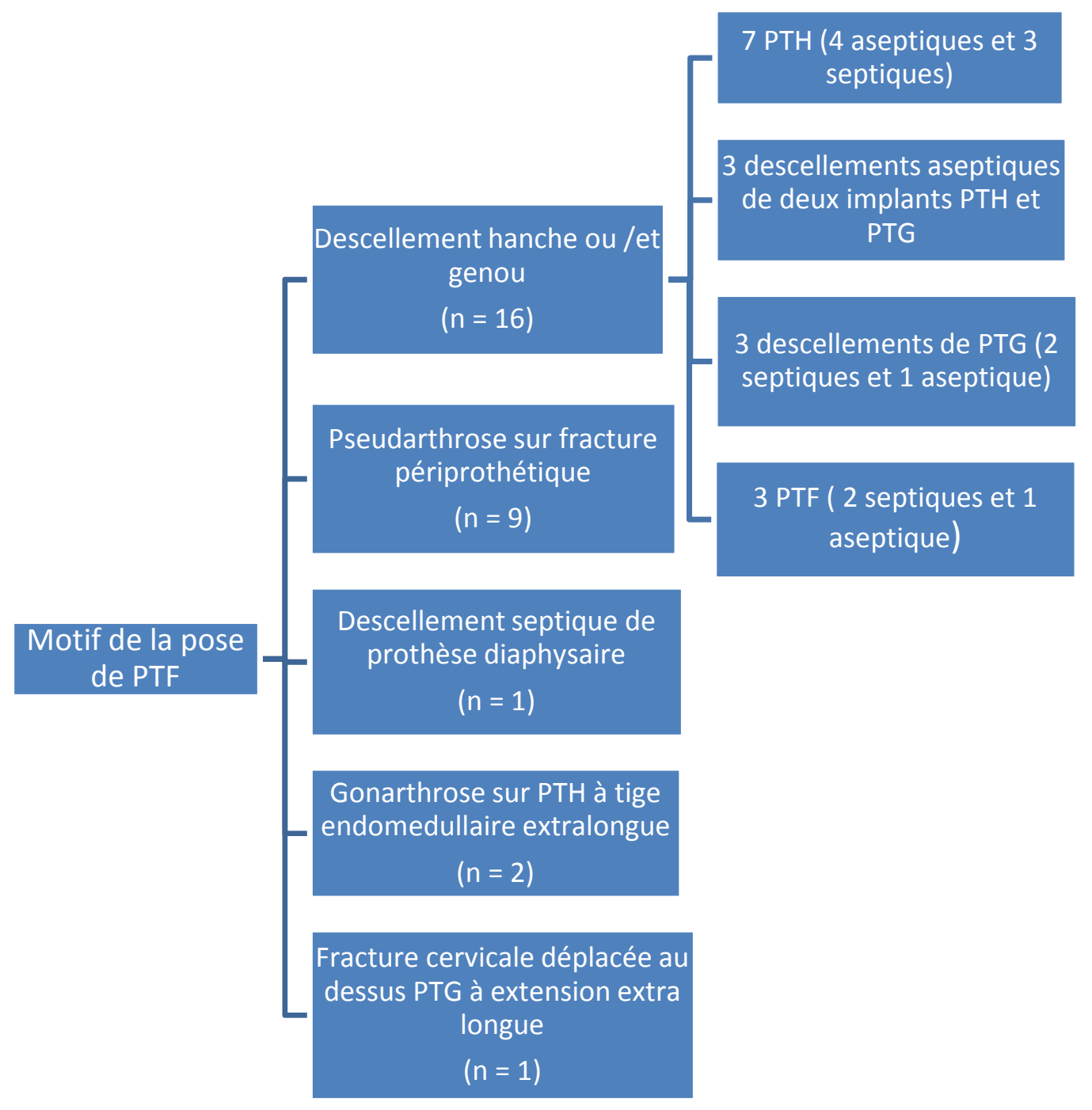




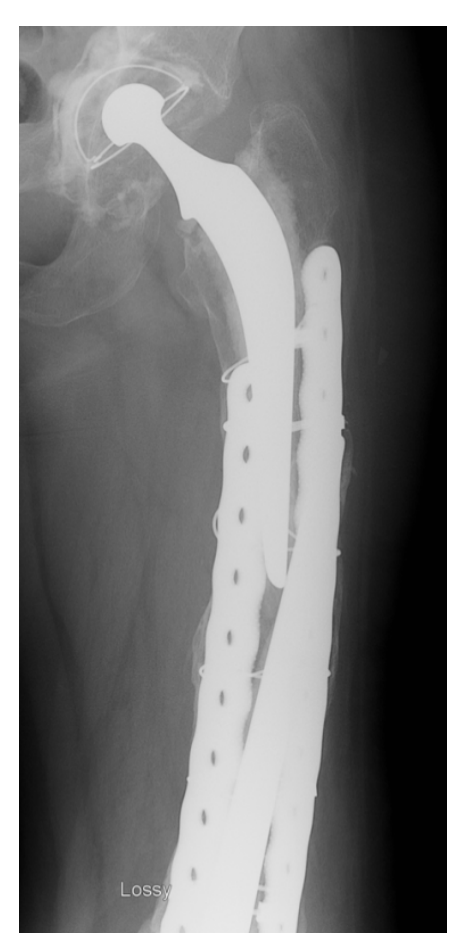

$4 \mathrm{~A}$

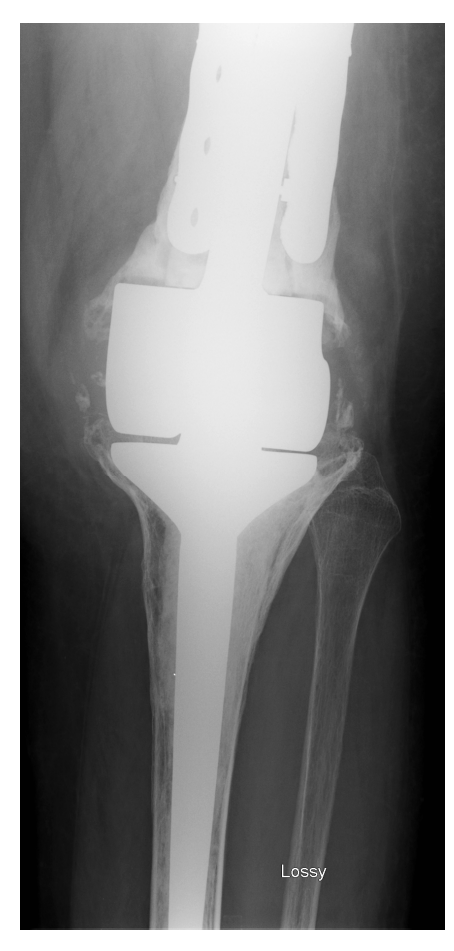

4B

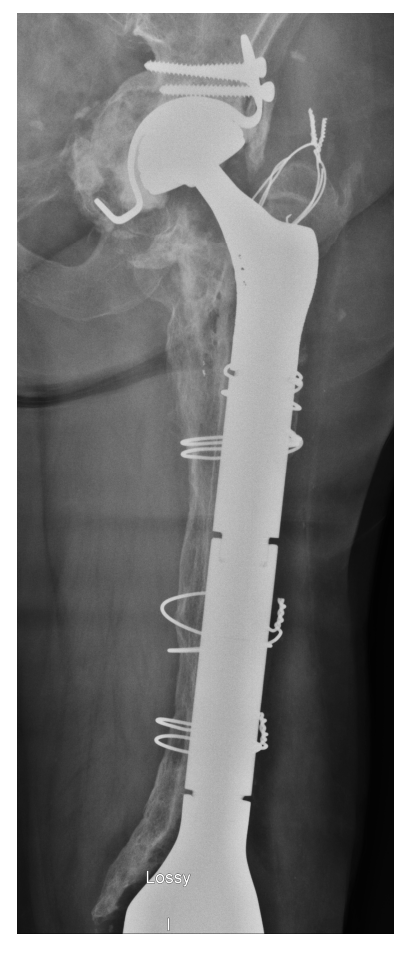

$4 \mathrm{C}$ 


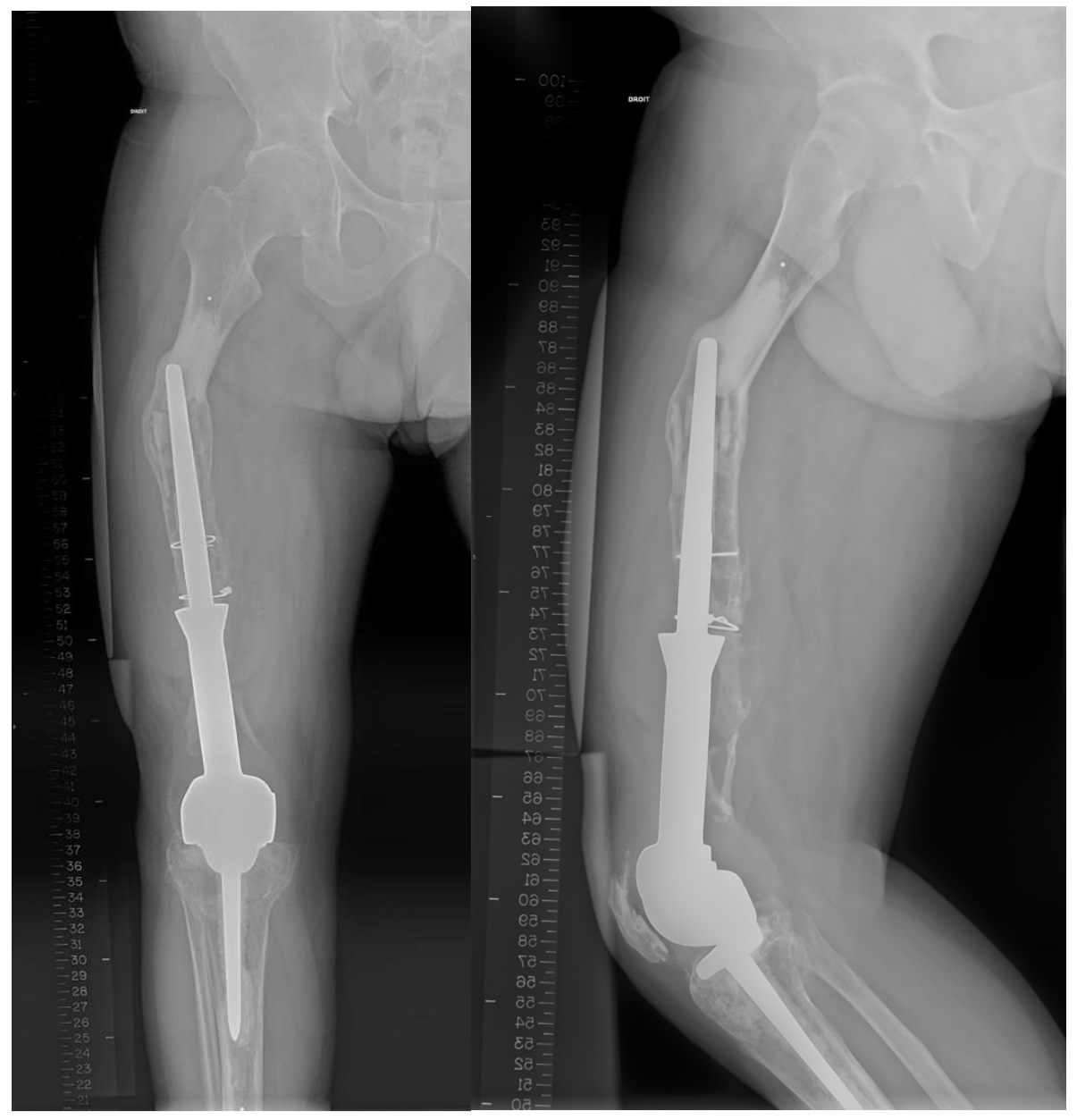

$5 A$

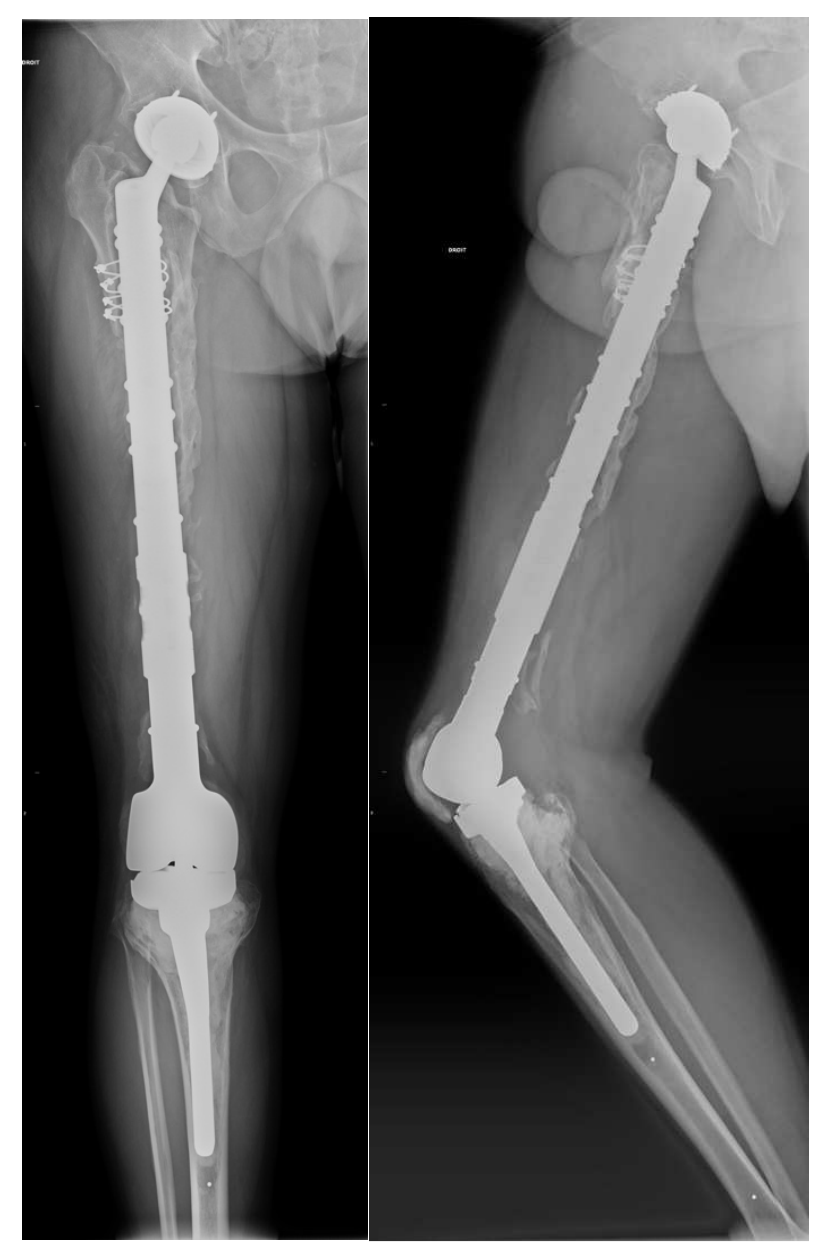

$5 B$ 